\title{
Dose-dense chemotherapy: ready for prime time
}

\author{
C. T. Dang, C. A. Hudis \\ Department of Medicine, Memorial Sloan-Kettering Cancer Center, New York, NY, USA.
}

\begin{abstract}
Breast cancer is one of the leading causes of cancer mortality in women in developed countries even though most women with the disease die of other causes. Postoperative adjuvant chemotherapy with anthracycline-based regimens has been proven to decrease the risk of relapse and cancer-related mortality in women with early stage breast cancer contributing to the improving survival seen with this disease. The taxanes have been effective additions to several adjuvant regimens based on recent prospective randomized studies. In the quest to further improve outcomes with adjuvant chemotherapy, dose and schedule modifications based on kinetic models have been compared with standard regimens. In this paper we review of several key trials testing a specific modification called 'dose-dense' chemotherapy.
\end{abstract}

Keywords: Adjuvant therapy; Breast cancer; Dose-dense chemotherapy

\section{Introduction}

Breast cancer is the second leading cause of cancer mortality in women. The most important prognostic factor in survival or recurrence after potentially curative surgery is the number of involved axillary lymph nodes. The prognosis for patients with extensive axillary lymph nodes at the time of presentation with primary breast cancer is poor despite standard dose-adjuvant chemotherapy $[1,2]$. Nevertheless, systemic chemotherapy remains a critical component in the eradication of occult micrometastatic disease in the adjuvant setting. In an effort to improve efficacy of conventional chemotherapy, the Cancer and Leukemia Group B led a Phase III intergroup (CALGB trial 9741) [3] which was designed to test a mathematical model of tumor growth based on the Norton-Simon hypothesis [4]. This hypothesis, developed over 30 years ago, and the kinetic model derived from it, formed the basis for the concept of dose-density and sequential therapy, both tested in

Correspondence to: Chau T. Dang, MD, Memorial Sloan-Kettering Cancer Center, 1275 York Avenue, Howard 713, New York, NY 10021, USA. E-mail: dangc@mskcc.org; Tel: +1212 639 7940; Fax: +1212 7728441

Publication date 31/05/05

$\mathrm{BCO} / 76 / 2002 / \mathrm{FO}$
CALGB 9741. Overall, this important trial convincingly showed that shortening the time interval between each chemotherapy cycle while maintaining the same dose size resulted in a significant improvement in disease-free survival (DFS) and overall survival (OS) in patients with node-positive breast cancer without increase in toxicity. This result is highly relevant, of immediate practical implication, and changes the standard practice of breast cancer treatment in the adjuvant setting.

\section{Tumor cell growth kinetics}

In order to understand dose-density, one must appreciate the concept of dose-intensification. In an attempt to improve benefit of current chemotherapy in breast cancer, dose-intensification has been evaluated extensively over the last decade. The term doseintensity is formulated as body-size adjusted dose $\left(\mathrm{mg} / \mathrm{m}^{2}\right)$ divided by time (per week) [5]. The most widely used method of increasing dose-intensity is dose-escalation, which has been shown to be modestly successful for some drugs in some dose ranges [6]. The total impact of therapy could relate to the cell kill for each dose, the length of time drugs are administered, and the rate of tumor growth between 
each treatment. If so, then a fixed cell kill achieved at shorter time intervals should improve the overall impact of therapy, as it would allow less time and opportunity for the emergence and proliferation of surviving, drug-resistant cell clones. This concept is termed dose-density [4].

Another attempt to improve efficacy of chemotherapy is through the use of sequential noncross-resistant regimens to maximize cell kill in a heterogeneous group of tumor cells. To understand the importance of sequential therapy, we must revisit tumor cell growth kinetics. Human solid tumors do not exhibit an exponential growth, but instead follow a Gompertzian growth pattern in which the tumor volume doubling time is not constant but instead increases with increasing tumor size, up to a certain mass/volume [7]. Likewise, tumor regrowth after less-than-curative therapy may be quite rapid after each cycle of therapy so complete eradication of disease may be difficult [8]. The Skipper-Schabel-Wilcox model, also termed the logkill model, was the first significant proliferation model in clinical oncology [9]. By this model, enough cycles of enough drugs at high enough individual doses should be able to kill a high percentage, if not all, of the cells. Unfortunately, this has not been clinically proven to be true for breast cancer. One possibility is that most tumors are composed of heterogeneous cells, some of which are likely to be slowly growing clones that are resistant to drugs used. An alternative model, the Norton-Simon model, predicts that the best way to cure this heterogeneous mix of cells is to eradicate the numerically dominant, faster-growing cells first, followed by eradication of the more slowly growing, resistant cells [4]. This is termed sequential therapy, which has been proven to be clinically superior to alternating therapy [10].

\section{Results from CALGB trial 9741/INT C9741}

CALGB trial 9741 was built on the concepts of dosedensity and sequential therapy. This study enrolled 2005 women with node-positive breast cancer. In this trial patients were randomized to one of four arms of treatment: (I) sequential doxorubicin $(A) \times$ $4 \rightarrow$ paclitaxel $(T) \times 4 \rightarrow$ cyclophosphamide $(C) \times 4$ conventionally every 3 weeks, (II) sequential $A \times 4 \rightarrow$ $\mathrm{T} \times 4 \rightarrow \mathrm{C} \times 4$ every 2 weeks with filgrastim, (III) AC $\times 4 \rightarrow T \times 4$ every 3 weeks and (IV) AC $\times 4 \rightarrow$ $\mathrm{T} \times 4$ every 2 weeks with filgrastim. This trial used a $2 \times 2$ factorial design to answer two questions:

1. Is dose-dense scheduling superior to conventional scheduling of chemotherapy?

2. Is sequential treatment superior to concurrent combination chemotherapy?
This study was powered to detect a $33 \%$ difference in either DFS or OS. Over $98 \%$ of patients who were enrolled received protocol therapy. At a median follow-up of 36 months, 351 patients had relapsed or died, compared with 515 expected treatment failures. The DFS was significantly prolonged for the dose-dense arms (II and IV) compared to the conventional arms (I and III; risk ratio $(R R)=0.74$, $P=0.010)$, and OS (RR $=0.69, P=0.013)$. The 4 -year DFS was $82 \%$ for dose-dense regimens and $75 \%$ for conventional regimens $(95 \%$ confidence interval (Cl), $73.7 \%$ to $76.2 \%)$. The 3-year OS was $92 \%(95 \% \mathrm{Cl}, 91.7 \%$ to $92.3 \%)$ in dose-dense arms and $90 \%$ in conventional arms $(95 \% \mathrm{Cl}, 89.6 \%$ to $90.4 \%)$. The differences between dose-dense and conventional regimens were expected to increase over time. There was no difference in either DFS or OS between sequential and concurrent chemotherapy schedules. There was no interaction between dose-density and sequence.

There was less-frequent severe grade 4 granulocytopenia in those patients on dose-dense regimens vs. conventional regimens ( $6 \%$ vs. $33 \%, P<0.0001$ ). Overall only $3 \%$ of patients were hospitalized for febrile neutropenia. Grade 3/4 emesis was more frequent in concurrent regimens than for sequential regimens ( $7 \%$ vs. $3 \%, P=0.0002)$. About $13 \%$ of patients on dose-dense $A C \rightarrow T$ had at least one red blood cell transfusion vs. none in conventional, sequential $\mathrm{A} \rightarrow \mathrm{T} \rightarrow \mathrm{C}$ vs. $<4 \%$ in other two arms $(P=0.0002)$. This was secondary to a greater frequency of grade 2 anemia in the dosedense $A C \rightarrow T$ arm [11], and this problem may be averted with the use of erythropoietin or darbepoietin. Only a small percentage of the patients required dose delay or reductions. The 3-year incidence of acute myelogenous leukemia or myelodysplasia was $0.18 \%$, and the incidence of leukemia was not influenced by filgrastim. A provocative and unexpected finding is that the dose-dense regimens were associated with a significantly reduced incidence of contralateral breast cancer $(0.3 \%$ vs. $1.5 \%$, $P=0.0004)$. Overall, all treatment arms were well tolerated [3].

The design of this trial allows one to draw strong conceptual conclusions. All patients received the same number of drugs at the same cumulative dose for each arm of the trial. Presently, these results are consistent with the mathematical predictions that dose-dense chemotherapy would result in superior survival over conventional regimens. Also, sequential therapy that preserves dose-density would maintain efficacy. There was no adverse impact of uncoupling $A$ from $C$ (nor any benefit). Longer followup is awaited to confirm a sustained benefit for this dose-dense therapy. 


\section{What are the results of other dose-dense trials?}

Many trials have been reported in which a standard regimen is compared with a dose-dense treatment, but confounding factors can make their interpretation challenging with regards to the narrow concept of dose-density. For example, Green et al. reported exciting results of dose-dense weekly vs. 3-weekly paclitaxel followed by fluorouracil/doxorubicin/ cyclophosphamide (FAC) as neoadjuvant therapy in operable breast cancer at M.D. Anderson Cancer Center. In this study the rate of pathologic complete response $(\mathrm{pCR})$ of weekly dose-dense paclitaxel arm is more than doubled that of 3-weekly paclitaxel (28.8\% vs. $13.6 \%, P<0.01$ ) [12]; whether this will translate to survival benefit will require longer followup. It is worth noting that in-breast response does not necessarily translate into improved outcomes as noted by prior trials $[13,14]$. Seidman et al. recently reported results of CALGB 9840 in the metastatic setting demonstrating that weekly paclitaxel was again superior to standard 3-weekly paclitaxel, in terms of response rate and time to progression, thus confirming the benefit of weekly paclitaxel [15]. These two trials are comparisons of low-dose therapy against higher doses, more treatments against fewer, and shorter intervals against longer ones. Hence, while they are consistent with the hypothesis that dose-dense treatment is superior, they are not pure tests of the concept.

Other trials have tested dose-dense regimens against standard regimens but results may be challenging to interpret. For example, Untch et al. clearly demonstrated the superiority of neoadjuvant dosedense, sequential $\mathrm{E} \times 3 \rightarrow \mathrm{T} \times 3$ every 2 weeks with filgrastim compared to conventional 3-weekly ET $\times$ 4 in terms of pCR and breast conservation rate. However, in this trial dose size, cycle number, and intervals varied. While we may firmly believe that dose-escalation was not important (dose-dense arm received higher cumulative doses of each chemotherapy), it remains somewhat uncertain as to which variable contributed to the improved outcome [16].

Mobus et al. recently reported results of the Arbeitsgemeinschaft fuer Gynaekologische Onkologie trial comparing EC $\left(90 / 600 \mathrm{mg} / \mathrm{m}^{2}\right) \times 4 \rightarrow T$ $\left(175 \mathrm{mg} / \mathrm{m}^{2}\right) \times 4$ all 3 -weekly vs. dose-dense E $\quad\left(150 \mathrm{mg} / \mathrm{m}^{2}\right) \times 3 \rightarrow \mathrm{T} \quad\left(225 \mathrm{mg} / \mathrm{m}^{2}\right) \times 3 \rightarrow \mathrm{C}$ $\left(2500 \mathrm{mg} / \mathrm{m}^{2}\right) \times 3$ all 2-weekly with filgrastim. Overall, there was a statistically significant improvement in the 3-year relapse-free survival (94 vs. 127 events, $P=0.0009$ ) and OS (43 vs. 60 events, $P=0.03)$ in favor of dose-dense $\mathrm{E} \rightarrow \mathrm{T} \rightarrow \mathrm{C}$ [17]. Again, however, the multiple variables (different numbers of cycles and dose sizes for each drug) can prevent some from interpreting this as a pure trial testing the concept of dose-density. Yet the advantage for fewer cycles given more often using drugs without proven dose-to-response benefits in the range tested makes a very strong argument in favor of dose-dense scheduling.

Overall it is our firm belief that the advantage of the every other week treatment in these studies derives from the change in dose-density but these other factors could contribute. Hence these trials are supportive of - that is they are consistent with the hypothesis that dose-density matters.

Not all dose-dense trials were viewed as positive. A pure comparison of more vs. less dose-dense treatment was reported by Venturini et al. from the Gruppo Oncologico del Nord-Ovest group on the comparative efficacy of adjuvant standard vs. accelerated fluorouracil/epirubicin/cyclophosphamide (FEC), for patients with early breast carcinoma [18]. Patients were randomly assigned to receive six cycles of FEC60 (fluorouracil $600 \mathrm{mg} / \mathrm{m}^{2}$, epirubicin $60 \mathrm{mg} / \mathrm{m}^{2}$, and cyclophosphamide $600 \mathrm{mg} / \mathrm{m}^{2}$ ) given either every 3 weeks (FEC21) or every 2 weeks (FEC14) with filgrastim. After a median follow-up of 6.7 years, there was a trend in improved survival in favor of the FEC14 arm but this did not reach statistical significance (hazard ratio $(\mathrm{HR}) 0.82 ; 95 \% \mathrm{Cl}$ $0.6-1.12 ; P=0.22)$. The lack of a statistically significant benefit for dose-dense FEC60 in this trial could be due to the use of a substandard version of FEC ('60' as opposed to ' 100 ') but more importantly to the underlying biostatistical assumptions in the study. Here the investigators expected the control arm to have a significantly worse outcome than was actually observed. As a result, the study was underpowered to prove a difference between the arms. Although the Venturini trial was a pure test of dosedensity, any trend in benefit favoring FEC14 may never reach statistical significance and, at the same time, it is underpowered to prove equivalence.

Therasse et al. reported results of randomized Phase III trial by European Organization for Research and Treatment of Cancer (EORTC)/National Cancer Institute of Canada/Swiss Group for Clinical and Epidemiological Cancer Research comparing standard CEF $\times 6$ (oral cyclophosphamide days 1-14; epirubicin and fluorouracil on days 1 and 8 , every 28 days) to dose-intensified biweekly EC $\times 6$ with filgrastim (without 5-fluorouracil) as primary chemotherapy in locally advanced breast cancer. This trial did not show therapeutic benefit of dose-dense EC over conventional CEF [19]. However, the interpretability of this trial as one of dose-intensity or dosedensity is difficult given the inclusion of 5-fluorouracil in one arm, the different schedules of administration, and the variation in dose. On the other hand one can 
view this study with optimism in that similar efficacy was achieved with both treatments but duration of treatment was half as long with dose-dense EC without agents (5-FU) and additional toxicities. Jackisch et al. reported results of the Geparduo study of preoperative chemotherapy comparing sequential 3-weekly AC $\times 4 \rightarrow$ docetaxel (D) $\times 4$ vs. 2-weekly $A D \times 4$. In this trial $A C \rightarrow D$ was superior to dosedense $A D$ in terms of clinical response rate, $p C R$, pathologic node-negativity rate, and breast conservation rate. Again, the results of this trial should not be surprising as patients in $A C \rightarrow D$ received an additional drug (i.e. cyclophosphamide) and that the cumulative doses of $A$ and $D$ were higher than that of AD arm [20].

The National Cancer Institute of Canada MA.21 is currently comparing standard CEF $\times 6$ (oral cyclophosphamide days 1-14; epirubicin and fluorouracil on days 1 and 8 , every 28 days) vs. 3-weekly $\mathrm{AC} \times 4 \rightarrow \mathrm{T} \times 4$ vs. 2 -weekly $\mathrm{EC} \times 6 \rightarrow 3$-weekly $\mathrm{T} \times 4$ [21]. The EC is dose-dense but the other arms are 'standard'. Interpretation of this trial with regard specifically (and narrowly) to dose-density will be interesting as confounding factors include the use of 5 -fluorouracil, the doxorubicin as opposed to epirubicin, paclitaxel in two of the three arms among others.

\section{Where do we go from here?}

With the use of filgrastim, several piloted dosedense trials have demonstrated that 2-weekly chemotherapy is feasible [22-24]. CALGB 9741 showed powerfully not only the feasibility of this approach, but also the superiority of dose-dense over conventional chemotherapy. These findings are extremely exciting and are consistent with the mathematical predictions of that by shortening the time interval between each chemotherapy treatment could result in more effective eradication of malignant cells and thus result in improved survival. The sequential concept tested by CALGB 9741 failed to show superiority of single agent sequential therapy over combined $A C$ sequenced into $\mathrm{T}$, but showed no disadvantage for uncoupling agents from one another, either. This result challenges the conventional wisdom not of polychemotherapy, but specifically of combination chemotherapy, as a gold standard. Longer follow-up will illuminate further late recurrences, secondary tumors, and toxicities but this trial is pivotal and confirmatory studies are welcomed. Based on current data, practicing oncologists may consider treating breast cancer patients in this particular dose-dense fashion. However, one should apply appropriate caution in extrapolation of this data to all regimens outside of a clinical trial setting, as toxicities may emerge that are unexpected.
For instance, a pilot trial was conducted at Memorial Sloan-Kettering Cancer Center (MSKCC) showing that FEC100 every 2 weeks $\times 6$ with filgrastim was not feasible due to a $9 \%$ incidence of unexpected chemical pneumonitis [25].

Future directions should include confirmatory trials that involve the same number of drugs at the same doses as a negative result of a 2-drug regimen, albeit dose-dense, against a 3-drug regimen may be simply due to the lack of the additional drug $[19,20]$. Quality of life (QOL) should be measured, as it is important to determine if QOL can be maintained while trying to achieve DFS and OS benefit with dose-dense therapy. Instinctively, it is foreseeable that patients would be willing to endure the minor inconvenience of filgrastim to shorten duration of treatment and to gain therapeutically. Costeffectiveness analysis for the addition of filgrastim would be important to know. At MSKCC we have investigated an increased dose-dense schedule with a regimen $E C \times 4 \rightarrow T \times 4$ given every $10-11$ days with filgrastim, and results will be available soon [26]. With the availability of pegylated filgrastim (Neulasta, Amgen, Inc., Thousand Oaks, CA), a novel formulation that provides once-per-cycle dosing, as opposed to daily injection, it will be interesting to explore its usage in 2-weekly regimens. Burstein et al. recently reported that pegfilgrastim appears to be safe and effective in facilitating dose-dense $A C \rightarrow T$ in the adjuvant treatment of breast cancer [27]. Currently, at MSKCC we are piloting a trial of dose-dense $\mathrm{EC} \times 6 \rightarrow \mathrm{T} \times 6$ with pegylated filgrastim support in breast cancer patients and we are testing the safety of 'conventional' dose-dense AC followed by paclitaxel (as per C9741) given with trastuzumab. In this era of targeted therapy, future research should combine biologic therapies, including anti-angiogenic treatments to dose-dense regimens to continue this important incremental advance in adjuvant treatment.

\section{References}

1. Fisher B, Bauer M, Wickerham DL, et al. Relation of number of positive axillary nodes to the prognosis of patients with primary breast cancer: an NSABP update. Cancer 1983; 52: 1551-1557.

2. Saez RA, McGuire WL, Clark GM, et al. Prognostic factors in breast cancer. Sem Surg Oncol 1989; 5: 102-110.

3. Citron ML, Berry DA, Cirrincione C, et al. Randomized trial of dose-dense versus conventionally scheduled and sequential versus concurrent combination chemotherapy as postoperative adjuvant treatment of node-positive primary breast cancer: first report of Intergroup trial C9741/Cancer and Leukemia Group B Trial 9741. J Clin Oncol 2003; 21: 1431-1439.

4. Norton L, Simon R. The Norton-Simon hypothesis revisited. Cancer Treat Rep 1986; 70: 163-169. 
5. Hryniuk W, Levine MN. Analysis of dose intensity for adjuvant chemotherapy trials in stage II breast cancer. J Clin Oncol 1986; 4: 1162-1170.

6. Wood WC, Budman DR, Korzun AH, et al. Dose and dose intensity of adjuvant chemotherapy for stage II, node-positive breast carcinoma. New Engl J Med 1994; 330: 1253-1259.

7. Norton L. A Gompertzian model of human breast cancer growth. Cancer Res 1988; 48: 7067-7071.

8. Dang CT, Gilewski T, Surbone A, Norton L. Cytokinetics. In: Holland JF, Frei E, Bast RC, et al. (Eds). Cancer Medicine, 6th edition. Baltimore: Williams and Wilkins; 2003: 645-668.

9. Skipper HE. Laboratory models: the historical perspective. Cancer Treat Rep 1986; 70: 3-7.

10. Bonadonna G, Zambette M, Valagussa P. Sequential or alternating doxorubicin and CMF regimens in breast cancer with more than three positive nodes: 10 year results. J Am Med Assoc 1995; 273: 542-547.

11. Citron ML, Berry DA, Cirrincione C, et al. Dose-dense (DD) AC followed by paclitaxel is associated with more frequent moderate anemia compared to sequential (S) and/or less DD treatment: Update by CALGB on Breast Cancer Intergroup Trial C9741 with ECOG, SWOG, and NCCTG [Abstract 620]. Proc American Soc Clin Oncol 2005; 24: 33s.

12. Green MC, Buzdar AU, Smith S, et al. Weekly paclitaxel followed by FAC as primary systemic chemotherapy of operable breast cancer improves pathologic complete remission rates when compared to every 3-week $P$ therapy followed by FAC: final results of a prospective phase III randomized trial [Abstract 135]. Proc Am Soc Clin Oncol 2002; 21: 35a.

13. Heys SD, Hutcheon AW, Sarkar TK, et al. Neoadjuvant docetaxel in breast cancer: 3-year survival results from the Aberdeen trial. Clin Breast Cancer 2002; 3(Suppl 2): S69-S74.

14. Bear HD, Anderson S, Smith RE, et al. A randomized trial comparing preoperative doxorubicin/cyclophosphamide (AC) to preoperative AC followed by preoperative docetaxel $(T)$ and to preoperative AC followed by postoperative $T$ in patients with operable carcinoma of the breast: results of NSABP B-27 [Abstract 26]. Breast Cancer Res Treat 2004; 88(Suppl 1): S16.

15. Seidman AD, Berry D, Cirrincione C, et al. CALGB 9840: Phase II study of weekly $(\mathrm{W})$ paclitaxel $(\mathrm{P})$ via 1-hour (h) infusion versus standard (S) $3 \mathrm{~h}$ infusion every third week in the treatment of metastatic breast cancer (MBC), with trastuzumab (T) for HER2 positive MBC and randomized for T in HER2 normal MBC [Abstract 512]. Proc Am Soc Clin Oncol 2004; 23: 6s.

16. Untch M, Konecny $\mathrm{G}$, Ditsch $\mathrm{N}$, et al. Dose-dense sequential epirubicin-paclitaxel as preoperative treatment of breast cancer: results of a randomized trial AGO study [Abstract 133]. Proc Am Clin Oncol 2002; 21: 34a.

17. Mobus VJ, Untch M, Du Bois A, et al. Dose-dense sequential chemotherapy with epirubicin $€$, paclitaxel $(T)$ and cyclophosphamide (C) (ETC) is superior to conventional dosed chemotherapy in high-risk breast cancer patients $(\geqslant 4+L N)$. First results of an AGO-trial [Abstract 513]. Proc Am Soc Clin Oncol 2004; 23: 6s.

18. Venturini M, Aitini E, Del Mastro L, et al. Phase III adjuvant trial comparing standard versus accelerated FEC regimen in early breast cancer patients. Results from GONO-MIG1 study [Abstract 12]. Breast Cancer Res Treat 2003; 82: S9.

19. Therasse $P$, Mauriac L, Welnicka-Jaskiewicz $M$, et al. Final results of a randomized phase III trial comparing cyclophosphamide, epirubicin, and fluorouracil with doseintensified epirubicin and cyclophosphamide plus filgrastim as neoadjuvant treatment in locally advanced breast cancer. A EORTC-NCIC-SAKK multicenter study. $J$ Clin Oncol 2003; 21: 843-850.

20. Jackisch C, Von Minckwitz G, Rabb G, et al. Primary endpoint analysis of the Geparduo-study-preoperative chemotherapy (PCT) comparing dose-dense versus sequential Adriamycin/docetaxel combination in operable breast cancer [Abstract 152]. Breast Cancer Res Treat 2002; 76: S50.

21. Trudeau ME. Optimizing adjuvant breast cancer chemotherapy: rationale for MA.21 study. Oncology 2001; 15(Suppl 7): 7-13.

22. Hudis C, Seidman A, Baselga J, et al. Sequential dosedense doxorubicin, paclitaxel, and cyclophosphamide for respectable high-risk breast cancer: feasibility and efficacy. J Clin Oncol 1999; 17: 93-100.

23. Hudis C, Fornier M, Riccio L, et al. 5-year results of dose-intensive sequential adjuvant chemotherapy for women with high-risk node-positive breast cancer: a phase II study. J Clin Oncol 1999; 17: 1118-1126.

24. Fornier MN, Seidman AD, Theodoulou $M$, et al. Doxorubicin followed by sequential paclitaxel and cyclophosphamide versus concurrent paclitaxel and cyclophosphamide: 5-year results of a phase II randomized trial of adjuvant dose-dense chemotherapy for women with node-positive primary breast cancer. Clin Cancer Res 2001; 7: 3934-3941.

25. Dang CT, D'Andrea G, Moynahan M, et al. Phase II study of dose-dense 5-fluorouracil, epirubicin, and cyclophosphamide (FEC) followed by alternating weekly taxanes in high-risk, 4 or more node-positive breast cancer: feasibility. Clin Cancer Res 2004; 10: 5754-5761.

26. Fornier MN, Seidman AD, Lake D, et al. Increased dosedensity (DD) is feasible: a pilot study of epirubicin and cyclophosphamide (ECO followed by paclitaxel $(\mathrm{T})$ ), at a 10-11 day interval, with filgrastim support, for women with early breast carcinoma [Abstract 616]. Proc Am Soc Clin Oncol 2005; 24: 32s.

27. Burstein HJ, Parker LM, Doherty J, et al. Use of the longacting hematopoietic growth factors pegfilgrastim and darbepoietin alfa in support of dose-dense adjuvant chemotherapy [Abstract 1055]. Breast Cancer Res Treat 2004; 88: S60. 